\title{
Effect of breakfast omission and consumption on energy intake and physical activity in adolescent girls: a randomised controlled trial
}

\author{
Julia K. Zakrzewski-Fruer ${ }^{1 *}$, Tatiana Plekhanova ${ }^{1}$, Dafni Mandila ${ }^{2}$, Yannis Lekatis ${ }^{2}$ and Keith Tolfrey ${ }^{2}$ \\ ${ }^{1}$ School of Sport Science and Physical Activity, Institute for Sport and Physical Activity Research, University of Bedfordshire, \\ Bedford MK41 9EA, UK \\ ${ }^{2}$ Paediatric Exercise Physiology Research Group, School of Sport, Exercise and Health Sciences, Loughborough University, \\ Loughborough LE11 3TU, UK
}

(Submitted 7 April 2017 - Final revision received 14 July 2017 - Accepted 26 July 2017)

\section{Abstract}

It is not known if breakfast consumption is an effective intervention for altering daily energy balance in adolescents when compared with breakfast omission. This study examined the acute effect of breakfast consumption and omission on free-living energy intake (EI) and physical activity (PA) in adolescent girls. Using an acute randomised cross-over design, forty girls (age 13.3 (SD 0.8 ) years, BMI $21 \cdot 5$ (sD $5 \cdot 0$ ) $\mathrm{kg} / \mathrm{m}^{2}$ ) completed two, 3-d conditions in a randomised, counter-balanced order: no breakfast (NB) and standardised (approximately $1962 \mathrm{~kJ}$ ) breakfast (SB). Dietary intakes were assessed using food diaries combined with digital photographic records and PA was measured via accelerometry throughout each condition. Statistical analyses were completed using repeated-measures ANOVA. Post-breakfast EI was 483 (SD 1309) kJ/d higher in NB $v$. SB $(P=0.025)$, but total daily EI was 1479 (sD 11311$) \mathrm{kJ} / \mathrm{d}$ higher in SB $v$. NB $(P<0.0005)$. Daily carbohydrate, fibre and protein intakes were higher in $\mathrm{SB} v$. NB $(P<0 \cdot 0005)$, whereas daily fat intake was not different $(P=0 \cdot 405)$. Effect sizes met the minimum important difference of $\geq 0 \cdot 20$ for all significant effects. Breakfast manipulation did not affect post-breakfast macronutrient intakes $(P \geq 0.451)$ or time spent sedentary or in PA $(P \geq 0.657)$. In this sample of adolescent girls, breakfast omission increased post-breakfast free-living EI, but total daily EI was greater when a SB was consumed. We found no evidence that breakfast consumption induces compensatory changes in PA. Further experimental research is required to determine the effects of extended periods of breakfast manipulation in young people.

Key words: Breakfast: Children: Exercise: Health: Nutrition: Youth

There is a common belief that breakfast is the 'most important meal of the day' ${ }^{(1)}$. However, about one-third of young people, including children (pre-pubertal and typically $<11$ in girls and $<13$ years in boys) and adolescents (between puberty and adulthood $)^{(2)}$, in many countries skip breakfast regularly ${ }^{(3,4)}$. Cross-sectional reports that show infrequent breakfast consumption to be associated with overweight and obesity have led to premature assumptions that breakfast can be used as an intervention for weight loss ${ }^{(5)}$. Indeed, the lower adiposity status in children who frequently consume breakfast was not observed uniformly across twelve countries ${ }^{(4)}$. Thus, the strength, direction and causal nature of associations between breakfast frequency and adiposity are questionable.

The mechanistic basis for a causal link between breakfast frequency and adiposity may be examined by assessing energy intake (EI) and expenditure. Indeed, a sustained positive energy balance where EI exceeds energy expenditure causes weight gain $^{(6)}$. Despite their higher adiposity, young people who skip breakfast have lower ${ }^{(7,8)}$ or similar ${ }^{(9)}$ daily EI when compared with breakfast consumers. Interventions show that $1 \mathrm{~d}$ of breakfast omission did not increase subsequent EI to compensate for the energy deficit created by breakfast omission in children aged 8-10 years ${ }^{(10)}$ and in adolescents aged $13-17$ years ${ }^{(11)}$. Increased lunchtime EI has, however, been reported in men in response to $1 \mathrm{~d}$ of breakfast omission ${ }^{(12)}$. Under free-living conditions, adults generally show higher daily EI when breakfast is consumed $^{(13,14,15)}$, even when reductions in EI at lunch ${ }^{(15)}$ or between 12.00 and 18.00 hours $^{(13)}$ are observed. However, daily EI was similar in obese adults assigned to daily breakfast omission or consumption for 6 weeks ${ }^{(16)}$. In overweight and obese 'breakfast-skipping' females aged 15-20 years, daily EI was increased with normal-protein breakfast consumption, but a high-protein breakfast reduced evening snacking and did not increase daily $\mathrm{EI}^{(17)}$. In a similar mixed-sex sample, 12 weeks of high-protein breakfast consumption reduced free-living daily EI, whereas breakfast skipping and normal-protein breakfast

Abbreviations: $\mathrm{CHO}$, carbohydrate; EI, energy intake; FD, free-living diet; GI, glycaemic index; LoA, limits of agreement; LPA, light physical activity; NB, no breakfast; PA, physical activity; SB, standardised breakfast.

* Corresponding author: Dr J. K. Zakrzewski-Fruer, email Julia.Fruer@beds.ac.uk 
consumption did not ${ }^{(18)}$. However, these studies were based on young people accustomed to breakfast omission and only determined the impact of breakfast addition, not removal ${ }^{(17,18)}$.

When determining free-living EI via self-report in adolescents, compliance and underreporting are major challenges ${ }^{(19)}$. Adolescents report a preference for methods using technology, such as a disposable camera ${ }^{(19)}$, which eliminate the need for participants to estimate portion size and are less burdensome than weighed food diaries ${ }^{(20,21)}$. Digital photography methods have been validated against weighed food diaries and $24 \mathrm{~h}$ recall in adults ${ }^{(21)}$ and are reliable and valid when measuring children's food intake in cafeteria settings ${ }^{(22)}$. However, understanding the individual variation in free-living EI assessed using digital photography requires investigation to determine clinically meaningful intervention effects ${ }^{(23)}$

In addition to EI, physical activity (PA) is a key determinant of energy balance, weight gain and health ${ }^{(6,24)}$. Cross-sectional studies using objective measures of PA (e.g. accelerometry) have reported more frequent breakfast consumption to be associated with higher PA in girls but not boys ${ }^{(25)}$, or in boys but not girls ${ }^{(26)}$, or on weekends but not weekdays ${ }^{(27)}$. In lean ${ }^{(14)}$ and obese ${ }^{(16)}$ adults assigned to 6 weeks of daily breakfast consumption or omission, higher PA energy expenditure in the morning was shown in the breakfast consumption groups, and this resulted in increased total daily PA energy expenditure in the lean adults ${ }^{(14)}$. In support, an acute within-participant crossover study showed increased morning PA energy expenditure assessed via accelerometry when breakfast was consumed compared with when it was omitted in women ${ }^{(15)}$. However, another study using pedometers and heart rate monitors showed no effect ${ }^{(28)}$. Furthermore, it is not known whether consuming breakfast can increase PA in young people.

The adolescent period is a crucial time to promote dietary and PA behaviours for health, particularly in girls ${ }^{(29)}$. Furthermore, breakfast skipping is highly prevalent in this population ${ }^{(30)}$. Thus, the current study used a randomised, cross-over design to compare the effect of three consecutive weekdays of breakfast omission with standardised breakfast (SB) consumption on free-living EI and PA in girls aged 11-15 years. In a sub-sample, we examined the natural variability in free-living daily EI assessed using digital photography to determine the interindividual intervention response.

\section{Methods \\ Participants}

In this dual centre project, forty-nine girls aged 11-15 years were recruited from schools in the two locations in England. The study was conducted according to the guidelines laid down in the Declaration of Helsinki and all procedures involving human subjects/patients were approved by the respective University Research Ethics Committees. Written informed parental consent and child assent were obtained for all participants. Girls were excluded from the study if they had health related issues identified from a health screen questionnaire (e.g. allergies to the breakfast meals, fitted with a pacemaker) or were unable to walk or wear a PA monitor on their wrist.

\section{Preliminary measurements}

Stature was measured to the nearest $0.01 \mathrm{~m}$ using a portable Leicester height measure (SECA Corporation). Body mass was measured and percent body fat estimated to the nearest $0 \cdot 1 \mathrm{~kg}$ and $0 \cdot 1 \%$ respectively using a Tanita Body Composition Analyser (BC-418 MA; Tanita Corporation); subsequently, BMI was calculated as body mass divided by stature squared $\left(\mathrm{kg} / \mathrm{m}^{2}\right)$. Using age and sex-specific BMI centiles ${ }^{(31)}$, the girls were then classified as non-overweight (2nd to 85th centile) or overweight (85th to 95th centile). Waist circumference was measured to the nearest millimetre on exhalation at the midpoint between the last rib and top of the iliac crest using a non-elastic tape measure ${ }^{(32)}$. To describe the pubertal status of the study sample, the girls were asked to provide a validated ${ }^{(33,34)}$ self-assessment of their physical maturation using secondary sexual characteristics with the assistance of a primary home-based carer $^{(35)}$. Habitual breakfast frequency was assessed by asking participants the following question: 'How often do you usually have breakfast?' Participants were asked to indicate their response separately for weekdays and for weekend days. Response categories were 'never' to ' $5 \mathrm{~d}$ ' for the week, and 'never' to ' $2 \mathrm{~d}$ ' for the weekend. To provide an indication of the composition and energy content of the participants' habitual breakfasts, they recorded their breakfast intakes across $3 \mathrm{~d}$ (Tuesday, Wednesday and Thursday, i.e. the weekdays selected for the intervention described below) using digital photography and a written food diary. Breakfast EI and macronutrient intakes were calculated using Dietplan 6.7 (Forestfield Software).

\section{Experimental design}

Using a within-measures cross-over design, participants completed two, 3-d conditions in a counter-balanced order: no breakfast (NB) and SB. The conditions were conducted across the same three weekdays (i.e. Tuesday, Wednesday and Thursday) with either a 4- or an 11-d washout between conditions. For the duration of each 3 -d condition, participants were asked to record their diet and wear a wrist-worn accelerometer. The order of the conditions for each participant was produced using a computer-based random number generator by the principal investigator (J. K. Z.-F.). All data were collected between December 2013 and July 2014.

On each morning of each 3 -d condition, participants arrived at school in the fasted state (no food or drink consumed except water from 21.00 hours the previous day) and were asked not to eat breakfast (NB) or to consume the SB provided within $30 \mathrm{~min}$ (between 08.15 and 08.45 hours). For NB, participants were provided with $375 \mathrm{ml}$ of water. The participant's first opportunity to consume food or drink during the post-breakfast period was 10.30 hours (i.e. during school break time); thus, NB involved abstaining from energy-containing food and beverages between 21.00 hours the previous day and 10.30 hours the following morning. The participants were reminded on each day of the experimental conditions to refrain from snacking until 10.30 hours and reported that they complied with these instructions. The SB consisted of $56 \cdot 3 \mathrm{~g}$ wheat biscuits (Weetabix), $188 \mathrm{ml}$ semi-skimmed milk (Tesco Stores Ltd) and $375 \mathrm{ml}$ orange juice (Tesco Stores Ltd). The breakfast was low glycaemic index (GI), with a calculated GI of $54^{(36,37)}$. We chose 
a ready-to-eat cereal because this type of breakfast is associated with reduced obesity risk when compared with 'other breakfasts $^{\text {, }}{ }^{(7)}$. Recommendations suggest breakfast should be $20 \%$ of daily $\mathrm{EI}^{(38)}$ and reviews define breakfast typically as containing $20-35 \%$ of total daily energy needs ${ }^{(39)}$. Therefore, the SB contained $2092 \mathrm{~kJ}(500 \mathrm{kcal})$, equating to approximately $22-26 \%$ of daily energy requirements for 11-15-year-old girls, which take into account total daily energy expenditure plus the deposited energy costs for growth (8096-9594 kJ/d (1935-2293 kcal/d)) ${ }^{(40)}$. The SB was consumed at school rather than home to monitor compliance to the breakfast intervention and record any leftovers. If the SB was not consumed completely, it had to represent at least $20 \%$ of recommended daily EI for the participant to be included in the final sample ${ }^{(38)}$.

\section{Dietary assessment}

Participants recorded their daily diet using a digital camera (ViviCam 46; Vivitar) and food diary during each condition. A similar method has been validated previously in adults ${ }^{(21)}$ and children $^{(22)}$. The participants were asked to photograph all foods and beverages consumed and use the photographs as a recall method when completing their food diaries each evening. The food diaries included a record of the day, time, type, brand name, preparation method, estimated portion size and any leftovers of all food and drink consumed. Before completing the main conditions, the girls received a tutorial and written instructions on using the digital camera and food diary. On the morning after each day of dietary recording, the research team checked the participants' food diaries for completeness and cross-referenced the food diaries with the corresponding photographs. For missing photographs, portion size was estimated by the participants using the Young Person's Food Atlas ${ }^{(41,42)}$ with assistance from the research team; further details were added to the diary when appropriate. The mass of all foods and beverages consumed were estimated by comparing the digital photographs, taken by the participants, with the Young Person's Food Atlas ${ }^{(41,42)}$; this method has shown good agreement with weighed food diaries in children aged $\geq 11$ years $^{(43)}$. Food diaries were analysed using Dietplan 6.7 to estimate EI and macronutrient intakes, which were blocked into three time periods to separate breakfast, lunch and the evening meal and align with the school timetable: (i) 06.00-09.00 hours (including breakfast and early morning snacks), (ii) 10.3014.00 hours (including school break time snacks and lunch) and (iii) 14.00 until 21.00 hours (including dinner and evening snacks). Percentage breakfast EI compensation was calculated for SB relative to $\mathrm{NB}$ (i.e. the difference in post-breakfast $\mathrm{EI}$ between $\mathrm{SB}$ and NB divided by SB breakfast EI multiplied by 100); values of $100 \%$ indicated complete compensation for breakfast EI. Portions of fruit and vegetable consumed were quantified using the National Health Service guidelines for 5 portions $/ d^{(44)}$. High-fat and sugary snacks were defined as sweet baked products, cookies, ice cream, cakes, desserts, jams, sugar, sweets, nuts, potato crisps, cheese products, popcorn and soft drinks ${ }^{(8)}$.

\section{Variability of daily energy intake}

To determine the natural variability of daily EI, the primary outcome variable, a sub-sample of ten girls completed two, 3-d free-living diet (FD) conditions on 2 consecutive weeks at least 4 weeks before commencing the main study. On each day, the girls were free to eat and drink as they pleased and were instructed to record their dietary intakes using the digital photography and food diary method described above (see the 'Dietary assessment' section ). Thus, the data provided an indication of the variability in habitual dietary intakes between two 3-d periods rather than being an assessment of measurement reliability. The $95 \%$ limits of agreement (LoA) for daily EI were calculated by determining a 95\% limit above and below the mean difference for FD trial 1 and FD trial 2 (systematic error $\left(1.96 \times\right.$ random error)), as outlined by Bland \& Altman ${ }^{(45)}$. Student's paired $t$ tests were used to identify systematic change in the mean from trial one to two; whereas Pearson's product moment correlations between the paired residuals and the mean (proportional error check) and the absolute residuals and the mean (heteroscedasticity check) were examined to ensure the $95 \%$ LoA were representative of the whole sample. It has been estimated that excessive weight gain could be prevented in children and adolescents by reducing positive energy balance by $628 \mathrm{~kJ} / \mathrm{d}(150 \mathrm{kcal} / \mathrm{d})^{(46)}$. Therefore, we deemed that LoA of $\leq \pm 628 \mathrm{~kJ} / \mathrm{d}(150 \mathrm{kcal} / \mathrm{d})$ would be an acceptable testretest error for daily FD EI.

The data on variability of EI was also used as a control arm to quantify the true interindividual differences in the EI response to the breakfast intervention ${ }^{(23)}$. To determine a 'true' effect, Atkinson \& Batterham ${ }^{(23)}$ suggest comparing the sD of changes in the intervention arm (i.e., the effect SD) with the SD of changes from the control arm (i.e. the control SD). The SD of the true individual response is: $\sqrt{\left(S D_{\mathrm{e}}^{2}-S D_{\mathrm{C}}^{2}\right)}$, where $S D_{\mathrm{e}}$ is the effect $\mathrm{SD}$ and $S D_{\mathrm{C}}$ is the control $\mathrm{SD}^{(23)}$. The magnitude of the $\mathrm{SD}$ of true individual responses is appraised in terms of clinical importance. As with our LoA analysis, we deemed that a difference of $\geq \pm 628 \mathrm{~kJ} / \mathrm{d}(150 \mathrm{kcal} / \mathrm{d})$ would be clinically important when comparing the control and intervention $\mathrm{SD}^{(46)}$.

\section{Physical activity assessment}

Wrist-worn accelerometers that have been validated in 8-14 year olds (GENEActiv; Activinsights Ltd) were used to assess PA for the duration of each 3 -d condition ${ }^{(47)}$. The accelerometers were set to record at $85.7 \mathrm{~Hz}$ using a 1 -s epoch. The girls were asked to wear the accelerometers on their non-dominant wrist for $3 \mathrm{~d}$ at all times, removing only for bathing and water-based activities. To estimate daily time spent sedentary and in light PA (LPA) and moderate-to-vigorous PA (MVPA), GENEActiv cutpoints specific to 8-14 year olds were applied and expressed as percentage of total daily wear time ${ }^{(47)}$. The minimal amount of accelerometer data that were considered acceptable was $10 \mathrm{~h} / \mathrm{d}$ of wear time on all $3 \mathrm{~d}$ of both breakfast conditions ${ }^{(48)}$.

\section{Statistical analyses}

Statistical analyses were completed using IBM SPSS statistics software for Windows version 21 (IBM Corporation). Total daily EI, macronutient intakes and PA were compared between the two conditions using student's paired $t$ tests. For post-breakfast $\mathrm{EI}$ and macronutrient intakes, condition $\times$ time of day $(2 \times 2)$ repeated-measures ANOVA were used to examine differences 
between the conditions across the two time periods (i.e. 10.3014.00 and 14.00-21.00 hours). Weekday habitual breakfast frequency and BMI were considered as covariates, but were not used because the data did not satisfy the assumptions for covariate analysis (i.e. they were not significantly associated with the dependent variables across all conditions). Homogeneity of covariances were examined by Mauchly's test of sphericity, and a Greenhouse-Geisser correction was applied to the df if the sphericity assumption was violated. Cohen's d effect sizes $(d)$ were calculated to gauge the magnitude of differences between conditions for all significant effects. In the absence of published anchors, a $d \geq 0.20$ was considered the minimum important difference in all outcome measures, 0.50 to $<0.80$ moderate and $\geq 0.80$ large $^{(49)}$. Values are presented as means and standard deviations unless stated otherwise.

\section{Justification of sample size}

The calculated sample size was based on total daily EI (the primary outcome variable) and LPA (\% wear time), as this PA intensity has been shown to be sensitive to breakfast manipulation in adults ${ }^{(14)}$. A worthwhile difference in EI or energy expenditure between the two conditions was defined as $628 \mathrm{~kJ} / \mathrm{d}(150 \mathrm{kcal} / \mathrm{d})^{(46)}$. Our variability study showed that the $\mathrm{SD}$ of the EI difference between two, 3-d FD conditions was $1147 \mathrm{~kJ} / \mathrm{d}(274 \mathrm{kcal} / \mathrm{d})$. A $628 \mathrm{~kJ} / \mathrm{d}$ increase in energy expenditure requires approximately $45 \mathrm{~min}$ of $\mathrm{LPA}$ at $14.0 \mathrm{~kJ} / \mathrm{min}$ in adolescent girls ${ }^{(47,50)}$. This would equate to $5 \%$ of weekday waking hours and an SD of $6 \%{ }^{(48,51)}$. Using these figures, the number of participants estimated to detect a significant change at $90 \%$ power with a two-sided significance level of 0.05 was 35 for daily EI and 30 for LPA. To be included in the final sample, participants had to meet the following criteria: (1) attended the breakfast intervention club on all days; (2) abstain from all foods and beverages until 10.30 hours for NB; (3) consume at least $20 \%$ of recommended daily EI if they did not consume the SB completely ${ }^{(38)}$; (4) record their diet and wear the accelerometer as specified. Thus, forty-nine participants were recruited to allow for a dropout of $10-30 \%$.

\section{Results}

\section{Participant characteristics}

The final sample for dietary analysis included forty participants (nine were excluded: three broke the fast before 10.30 hours during NB, one did not consume an adequate amount of the SB and five did not record their dietary intakes as specified). Three participants that were included in the final sample did not eat all of the SB, but consumed enough so that SB EI was at least $20 \%$ of recommended daily $\mathrm{EI}^{(38)}$. Table 1 shows the anthropometric characteristics and habitual breakfast frequencies of the final sample. The nine girls who were excluded from the final analyses did not have significantly different physical characteristics or breakfast frequencies compared with the 40 who were included $(P \geq 0 \cdot 10)$.
Table 1. Participant characteristics

\begin{tabular}{|c|c|c|}
\hline & Average & Variability \\
\hline Age (years)* & $13 \cdot 3$ & 0.8 \\
\hline Stature $(m)^{*}$ & 1.60 & 0.08 \\
\hline Body mass $(\mathrm{kg})^{*}$ & $55 \cdot 2$ & $15 \cdot 4$ \\
\hline Body fat $\%{ }^{*}$ & $30 \cdot 7$ & $10 \cdot 1$ \\
\hline Waist circumference $(\mathrm{cm})^{*}$ & $71 \cdot 1$ & $12 \cdot 8$ \\
\hline BMI $\left(\mathrm{kg} / \mathrm{m}^{2}\right)^{*}$ & $21 \cdot 5$ & $5 \cdot 0$ \\
\hline Breast development† & 4 & 1 \\
\hline Pubic hair & 4 & 1 \\
\hline Weekday habitual breakfast frequency (d/week)* & $3 \cdot 7$ & 1.6 \\
\hline Weekend habitual breakfast frequency (d/week)* & $1 \cdot 7$ & 0.5 \\
\hline
\end{tabular}

${ }^{*}$ Means and standard deviations.

$\dagger$ Medians and interquartile ranges.

Variability of daily energy intake

At the group level ( $n$ 10), EI was similar between FD trial 1 and FD trial 2 (5063 (SD 1332) v. $5244(\mathrm{sD} 1293) \mathrm{kJ} / \mathrm{d}(1211$ (SD 319) $v$. $1255(\mathrm{sD} 309) \mathrm{kcal} / \mathrm{d}) ; t=-0.500 ; P=0.629)$. The systematic bias was 181 (random error 1147) kJ/d (43 (random error $274) \mathrm{kcal} / \mathrm{d}$ ). This resulted in $95 \% \mathrm{LoA}$ of -2067 to $2428 \mathrm{~kJ} / \mathrm{d}$, $(-494$ to $580 \mathrm{kcal} / \mathrm{d}$ ) (see online Supplementary Document S1). Significant proportional bias was not evident $(r-0 \cdot 038$; $P=0.917)$ and random errors were homoscedastic $(r-0.040$; $P=0 \cdot 912)$. The LoA based on all ten girls exceeded our $a$ priori acceptable test-retest variability of $\pm 628 \mathrm{~kJ} / \mathrm{d}(150 \mathrm{kcal} / \mathrm{d})$. However, seven of the ten participants had paired EI values across the repeat measurements that were within $\pm 628 \mathrm{~kJ} / \mathrm{d}$ $(150 \mathrm{kcal} / \mathrm{d})$, suggesting the natural variation in FD may be small enough to detect subtle changes in EI that could prevent excessive weight gain ${ }^{(46)}$. In the remaining three girls, one had a particularly large difference of $3141 \mathrm{~kJ} / \mathrm{d}$ between the repeat measurements. When excluding this participant $(n 9)$, the systematic error was reduced by -148 (random error 512 ) $\mathrm{kJ} / \mathrm{d}$, and the $95 \%$ LoA were tightened to -1151 to $855 \mathrm{~kJ} / \mathrm{d}$, but still exceed the $\pm 628 \mathrm{~kJ} / \mathrm{d}$ cut-off.

\section{Breakfast energy and macronutrient intake}

Accounting for leftovers, breakfast energy and macronutrient intakes for SB were: EI 1962 (sD 121) kJ (469 (sD 29) kcal), 88.3 (sD 6.0) g carbohydrate (CHO), 16.4 (sD 1.3) g protein, 5.7 (SD $0.7) \mathrm{g}$ fat and 5.8 (SD 0.6) $\mathrm{g}$ fibre. For comparison, the girls consumed 766 (SD 439) kJ (183 (sD 105) kcal), 29.4 (sD 16.1) g $\mathrm{CHO}, 5.4$ (SD 3.7) g protein, 5.4 (SD 4.5) g fat and 1.2 (SD 1.0) g fibre for breakfast habitually. The energy, $\mathrm{CHO}$, protein and fibre intake of the SB were higher than the girls habitual breakfasts $(P<0.005 ; d=3.69-5.32)$, whereas fat intakes were similar $(P=0 \cdot 672 ; d=0 \cdot 09)$.

\section{Daily and post-breakfast energy intake}

Fig. 1 shows daily and time-specific EI for each breakfast condition. Daily EI was higher in SB than NB $(P<0.0005)$. For daily EI, we quantified the true interindividual differences in the intervention responses using the control SD from the variability data described above and the sD of the residuals from the breakfast conditions ${ }^{(23)}$. The SD of the true individual response 


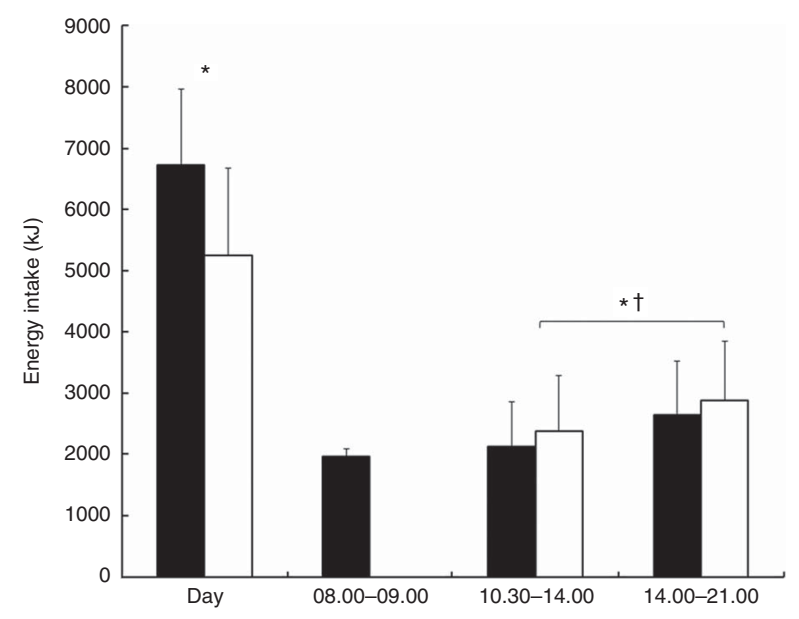

Fig. 1. Energy intake during different times of the day for the no breakfast (NB, $\square$ ) and the standardised breakfast (SB, $\square$ ) conditions ( $n$ 40). Values are means, with standard deviations represented by vertical bars. * Significant main effect of condition for total daily energy intake using paired $t$ tests and for total energy intake between 10.30 and 21.00 hours using a condition $\times$ time of day ANOVA $(P \leq 0.025)$. † Significant main effect of time of day using ANOVA $(P=0.003)$.

was $636(95 \%$ CI 229, 1042) kJ/d $(152(55,249) \mathrm{kcal} / \mathrm{d})$ for SB $v$. NB. Using our cut-off of $\geq 628 \mathrm{~kJ} / \mathrm{d}(150 \mathrm{kcal} / \mathrm{d})$, the large differences in the control and intervention SD may be clinically important.

Post-breakfast (i.e. 10.30-21.00 hours) EI was 483 (sD 1309$) \mathrm{kJ} / \mathrm{d}$ higher in NB compared with SB $(P=0 \cdot 025 ; d=0 \cdot 37)$, independent of the time of day $(P=0.993)$ (Fig. 1). The higher postbreakfast $\mathrm{EI}$ in NB accounted for 24 (SD 66) \% of the SB EI.

\section{Daily and post-breakfast macronutrient intakes}

Table 2 shows daily macronutrient intakes for each breakfast condition. Daily $\mathrm{CHO}$, fibre and protein intakes were higher in SB compared with NB ( $d \geq 0 \cdot 81$ for all comparisons), whereas daily fat intake was not. The effect of condition was NS for $\mathrm{CHO}$, fat, protein and fibre intakes for the post-breakfast period $(P \geq 0.451 ; d \leq 0.14$ for all comparisons) and there was no interaction with time of day $(P \geq 0.329)$. The time of day main effect showed that protein and fibre intakes were higher in the 14.00-21.00 hours period than the 10.30-14.00 hours period ( $P \leq 0.026 ; d \geq 0.50)$, but this difference only approached significance for $\mathrm{CHO}$ with a small effect $(P=0.054 ; d=0.40)$.

\section{Daily fruit and vegetable and high-fat and sugary snack consumption}

The breakfast condition main effect for daily portions of fruit and vegetables consumed was not significant (1.1 (SD 1.1) for NB $v \cdot 1 \cdot 1$ (SD $0 \cdot 8$ ) for $\mathrm{SB} ; P=0 \cdot 801$ ). Although the mean number of high-fat and sugary snacks consumed per day tended to be higher for $\mathrm{NB}(3.0$ (SD 1.5)) compared with SB (2.6 (SD 1.3)) $(P=0.097)$, the effect was only small $(d=0 \cdot 26)$.

\section{Daily physical activity}

A total of thirty-five girls had valid accelerometer data and were included in PA analyses. Wear time was 14.2 (sD 1.2) h/d for SB
Table 2. Daily energy and macronutrient intakes during $3 \mathrm{~d}$ of no breakfast (NB) or standardised breakfast consumption (SB) using a randomised cross-over design $(n 40)^{*}$ (Mean values and standard deviations)

\begin{tabular}{|c|c|c|c|c|c|c|}
\hline & \multicolumn{2}{|c|}{ SB } & \multicolumn{2}{|c|}{ NB } & \multirow[b]{2}{*}{$P$} & \multirow[b]{2}{*}{$d$} \\
\hline & Mean & SD & Mean & SD & & \\
\hline Energy (kJ/d) & 6728 & 1234 & 5249 & 1419 & $<0.0005$ & $1 \cdot 11$ \\
\hline Carbohydrate (g/d) & $243 \cdot 1$ & $50 \cdot 6$ & $158 \cdot 4$ & $50 \cdot 0$ & $<0.0005$ & 1.68 \\
\hline Fat $(g / d)$ & $51 \cdot 3$ & $18 \cdot 3$ & $48 \cdot 3$ & $17 \cdot 2$ & 0.405 & 0.17 \\
\hline Protein (g/d) & 61.4 & $18 \cdot 6$ & $47 \cdot 6$ & $15 \cdot 4$ & $<0.0005$ & 0.81 \\
\hline Fibre $(\mathrm{g} / \mathrm{d})$ & $13 \cdot 1$ & $3 \cdot 1$ & $7 \cdot 5$ & $2 \cdot 8$ & $<0.0005$ & 1.91 \\
\hline
\end{tabular}

d, Cohen's $d$ effect size.

* Paired $t$ tests and Cohen's $d$ effect sizes were used to compare the SB and NB conditions.

Table 3. Daily time spent sedentary and in physical activity during $3 \mathrm{~d}$ of no breakfast (NB) or standardised breakfast consumption (SB) using a randomised cross-over design $(n 35)^{*}$

(Mean values and standard deviations)

\begin{tabular}{lrrrrrrr}
\hline & \multicolumn{2}{c}{ SB } & & \multicolumn{2}{c}{ NB } & & \\
\cline { 2 - 3 } & Mean & SD & & Mean & SD & $P$ & $d$ \\
\hline Sedentary (\%WT) & 70.1 & 4.9 & 69.7 & 4.6 & 0.769 & 0.04 \\
LPA (\%WT) & 23.1 & 2.8 & & 23.2 & 2.8 & 0.657 & 0.06 \\
MVPA (\%WT) & 6.8 & 2.8 & 7.1 & 2.5 & 0.936 & 0.01 \\
\hline
\end{tabular}

$d$, Cohen's $d$ effect size; \%WT, percentage of total wear time; LPA, light physical activity; MVPA, moderate to vigorous physical activity.

* Paired $t$ tests and Cohen's $d$ effect sizes were used to compare the SB and NB conditions.

and $14 \cdot 1(\mathrm{sD} 1.3) \mathrm{h} / \mathrm{d}$ for $\mathrm{NB}(P=0 \cdot 488)$. Daily time spent sedentary or in LPA or MVPA (\% wear time) was not different between conditions (Table 3).

\section{Discussion}

Using an experimental cross-over design, this study showed that total daily EI was higher when adolescent girls consumed an approximately $1962 \mathrm{~kJ}$ SB when compared with NB over 3 consecutive weekdays. Although NB increased post-breakfast EI, the degree of EI compensation was small and only accounted for approximately $24 \%$ of the SB. In addition, breakfast manipulation did not affect time spent sedentary or in PA.

Our study supports previous research showing that breakfast consumption results in higher daily EI in young people ${ }^{(10,17)}$ and adults ${ }^{(12,13,14,15,52)}$ when compared with breakfast omission. In addition to being statistically significant, random withinsubject variation and measurement error did not explain the higher daily EI with breakfast consumption, which exceeded the natural variability in EI by more than $628 \mathrm{~kJ} / \mathrm{d}(150 \mathrm{kcal} / \mathrm{d})$ and may thus have clinical importance for weight gain ${ }^{(23,46)}$. The $483 \mathrm{~kJ} / \mathrm{d}(115 \mathrm{kcal} / \mathrm{d})$ increase in post-breakfast EI (i.e. between 10.30 and 21.00 hours) when the girls omitted breakfast amounted to only a quarter of the SB. This incomplete EI compensation may be due to the large size of the SB, which contained 2.6 times more energy than the participants' habitual breakfasts. As $\mathrm{CHO}$-based breakfasts containing approximately 
1.3 times less energy than our SB also did not result in EI compensation in young people $\mathrm{e}^{(10,17)}$, examining the effects of smaller breakfasts similar in energy content to the habitual breakfasts of adolescent girls (approximately $766 \mathrm{~kJ}$ in our sample) may enhance the ecological validity of the findings and likelihood EI compensation being complete. Although the increased EI with breakfast omission was distributed evenly across the day in our study, reports in adults indicate that these effects are specific to certain time periods ${ }^{(12,13,15)}$. It has also been shown that such effects may depend on the sex and breakfast habits of the sample ${ }^{(28)}$, whereas habitual breakfast frequency was not related to the outcome variables in our sample of adolescent girls. Nevertheless, the $95 \% \mathrm{CI}$ for the sD of the individual response (229-1042 kJ/d) indicates large interindividual variability in compensatory EI responses to breakfast omission. Thus, individual characteristics that may explain this variability, such as eating and PA habits, body composition, age and socioeconomic status warrant clarification.

Unlike most previous experimental studies in adults that involved unstandardised breakfast manipulation ${ }^{(13,14,16,28)}$, we provided a standardised, wholegrain ready-to-eat cereal-based breakfast. This type of breakfast was chosen because it has strong association with lowered obesity risk in adolescents ${ }^{(7)}$ and it is a convenient breakfast choice that requires minimal preparation time, which could be appealing for adolescents who skip breakfast due to a lack of time, ${ }^{, 53)}$. The small, but statistically significant increase in post-breakfast EI (i.e. between 10.30 and 21.00 hours) with breakfast omission reported here is in contrast with research in 'breakfast-skipping' adolescents showing that normal-protein breakfasts do not reduce subsequent $\mathrm{EI}^{(11,17,18)}$. Although breakfast GI was not reported in these studies ${ }^{(11,17,18)}$, it is possible that the low GI breakfast in our study promoted a slower release of glucose into the blood, which can reduce hunger and lunchtime $\mathrm{EI}^{(54)}$ and prolong satiety $^{(55)}$ in young people and may explain our discrepant findings ${ }^{(11,17,18)}$. However, the link between GI and EI is controversial $^{(56,57)}$, with the satiating effect of low GI foods possibly being due to their higher fibre content ${ }^{(56)}$. Nevertheless, the $5.8 \mathrm{~g}$ of fibre in our SB was within the range of the breakfasts in previous studies $(2 \cdot 0-6 \cdot 1 \mathrm{~g})^{(11,17)}$. Thus, differences in fibre content of the CHO-based breakfasts may not explain discrepancies between our results and previous work. Increased EI in response to breakfast omission may be due to a host of metabolic and behavioural responses induced by the appetite regulatory system, including increased pre-dinnertime neural activation in brain regions controlling food motivation/reward in late adolescent girls ${ }^{(17)}$. Such mechanisms require further research in young people.

Although weighed food records are often considered the criterion reference measure of free-living EI in adults, selfreported EI can be underestimated through poor compliance, participant selection bias, recording bias and changes to diet to facilitate recording ${ }^{(58)}$. In adolescents, the reliability and validity of weighed food diaries is less certain and the participant burden is particularly high ${ }^{(59,60)}$. In an attempt to increase compliance to recording dietary intakes in our study, we used a food diary accompanied by photographic evidence rather than a weighed food diary ${ }^{(19)}$. Although previous research has indicated that the addition of photographs to a traditional diet diary can enhance the validity and reliability of dietary recording ${ }^{(61)}$, our comparison of two 3 -d records showed $95 \%$ LoA for EI of -2067 to $+2428 \mathrm{~kJ} / \mathrm{d}(-494$ to $+580 \mathrm{kcal} / \mathrm{d})$. This high variability of free-living EI, potentially resulting from environmental, biological and methodological factors ${ }^{(62)}$, may limit the potential to detect clinically meaningful differences of $628 \mathrm{~kJ} / \mathrm{d}(150 \mathrm{kcal} / \mathrm{d})^{(46)}$. Previous literature on free-living EI variability using $3 \mathrm{~d}$ diet records has used varied statistical approaches and produced mixed findings ${ }^{(63,64)}$. Interestingly, our LoA are narrower than studies reporting 'acceptable' agreement with a 3 -d diet record in adults ${ }^{(65)}$ and a food menu in free-living young people ${ }^{(66)}$. Thus, the use of a clinically relevant anchor may have affected the interpretation of the LoA in these studies.

Consistent with cross-sectional reports ${ }^{(8,9,30)}$, the higher daily EI with SB consumption was due to higher intakes of $\mathrm{CHO}$, protein and fibre, whereas breakfast did not affect daily fat intakes. These differences in daily macronutrient intakes were a direct effect of the breakfast meal rather than post-breakfast intakes. Nevertheless, it is likely that the tendency for higher high-fat and sugary snack consumption contributed to the increased post-breakfast EI when breakfast was omitted. This finding also suggests that the nature of the cross-sectional association between infrequent breakfast consumption and higher unhealthy snack consumption ${ }^{(8,9)}$ may be causal. Overall, the girls consumed about one portion of fruit and vegetables a day, a concerning number considering that a minimum of five portions per day is recommended ${ }^{(44)}$. Although breakfast consumption has been associated with higher fruit and vegetable consumption ${ }^{(67,68)}$, the present study suggests that any such relations are not causal, at least in our sample over a 3-d intervention. As food groups other than fruit and vegetables contribute to micronutrient intakes, whether breakfast manipulation affects micronutrient intakes warrants examination using assessment periods of more than $3 \mathrm{~d}^{(69)}$.

The small post-breakfast EI compensation in the present study suggests that a higher PA energy expenditure may be more important in contributing to the healthy weight status in frequent breakfast consumers ${ }^{(1,3,4,5,7)}$. However, our finding that breakfast did not affect sedentary time or MVPA supports cross-sectional findings in girls aged 9-10 years when using accelerometry to quantify PA on weekdays and weekends ${ }^{(26)}$ and experimental research in adults showing no effect of breakfast manipulation on PA assessed via pedometers and heart rate monitors during a working week ${ }^{(28)}$. Although breakfast manipulation did not affect LPA in the girls in the present study, energy expenditure from LPA assessed over $7 \mathrm{~d}$ was higher in lean adults who consumed breakfast daily compared with those who omitted breakfast daily ${ }^{(14)}$. In the obese cohort of this 6 weeks intervention, total PA energy expenditure in the morning was higher in the breakfast group compared with the breakfast omission group ${ }^{(16)}$. An acute randomised cross-over trial using accelerometry also showed that consuming breakfast increased PA energy expenditure when compared with breakfast omission in women classified as habitual breakfast eaters ${ }^{(15)}$. The adolescent girls in our study may have responded differently to the adults in previous studies ${ }^{(14,15,16)}$ 
because the provision of breakfast at school meant that they had limited opportunity to engage in free-living PA directly after consuming breakfast. Thus, providing breakfast at home and including weekend days may increase the scope for detecting effects on PA.

The present study has several limitations. First, breakfast manipulation over 3 weekdays does not allow us to apply the findings to weekends, where diet and PA patterns are different ${ }^{(27,51,70)}$, or to determine the effects of longer intervention periods. Second, similar to previous studies ${ }^{(17,18)}$, we provided a fixed absolute breakfast portion. However, providing breakfast relative to daily energy requirements may be recommended to reduce between-participant variability in the response to breakfast manipulation. Similarly, methods that provide less variable measures EI would help support our findings, although this is challenging in free-living conditions ${ }^{(58,59,60)}$. In addition, standardising pre-intervention diet and the duration of the washout period between participants would help to minimise the influence of these factors on the study outcomes. Third, future studies employing more sensitive measures to quantify free-living PA or energy expenditure (e.g. combined heart rate-accelerometry or doubly labelled water) over longer measurement periods (e.g. 7 d) would be valuable in extending the findings reported here. In doing so, the possibility that breakfast consumption may affect PA through an interaction with wake time and sleeping patterns requires consideration. Differences in wake time are unlikely to have confounded the comparison between SB and NB in our study, as the provision of breakfast at school rather than at home meant that the participants were not required to wake up any earlier to consume the SB. Nevertheless, the independent effects of breakfast frequency, timing and composition warrant study. Finally, the generalisability of our findings to adolescent boys and to younger children requires investigation.

In conclusion, adolescent girls showed a small increase in post-breakfast EI of $483 \mathrm{~kJ} / \mathrm{d}(115 \mathrm{kcal} / \mathrm{d})$ that was not sufficient to compensate completely for 3 consecutive weekdays of breakfast omission when compared with SB consumption (approximately $1962 \mathrm{~kJ} / \mathrm{d}$ ). Thus, total daily EI remained greater when a SB was consumed. We also report no evidence of breakfast affecting time spent sedentary or in PA. These findings require examination using extended periods of breakfast manipulation and more sensitive devices to quantify PA energy expenditure in young people.

\section{Acknowledgements}

The authors thank Daniella Gibson, Shakyla Horton, Nicola King and Kelly Mason for their assistance with data collection for the study. This study received no external funding.

J. K. Z.-F. and K. T. designed the study (project conception, development of overall research plan, and study oversight). All authors contributed to the data collection (hands-on conduct of the experiments and data collection). J. K. Z.-F., T. P. and K. T. analysed the data and/or performed statistical analyses. J. K. Z.-F. and K. T. wrote the paper. J. K. Z.-F. had primary responsibility for final content. All authors have read and approved the final manuscript.

None of the authors has any conflicts of interest to declare.

\section{Supplementary material}

For supplementary material/s referred to in this article, please visit https://doi.org/10.1017/S0007114517002148

\section{References}

1. Rampersaud GC, Pereira MA, Girard BL, et al. (2005) Breakfast habits, nutritional status, body weight, and academic performance in children and adolescents. J Am Diet Assoc 105, 743-760.

2. Tanner JM, Whitehouse RH, Marshall WA, et al. (1975) Prediction of adult height, bone age, and occurrence of menarche, at age 4 to 16 with allowance for midparental height. Arch Dis Child 50, 14-26.

3. Vereecken C, Dupuy M, Rasmussen M, et al. (2009) Breakfast consumption and its socio-demographic and lifestyle correlates in schoolchildren in 41 countries participating in the HBSC study. Int J Public Health 54, Suppl. 2, S180-S190.

4. Zakrzewski JK, Gillison FB, Cumming S, et al. (2015) Associations between breakfast frequency and adiposity indicators in children from 12 countries. Int J Obes 5, Suppl. 2, S80-S88.

5. Brown AW, Bohan Brown MM \& Allison DB (2013) Belief beyond the evidence: using the proposed effect of breakfast on obesity to show 2 practices that distort scientific evidence. Am J Clin Nutr 98, 1298-1308.

6. Hill JO (2006) Understanding and addressing the epidemic of obesity: an energy balance perspective. Endocr Rev 27, 750-761.

7. Deshmukh-Taskar PR, Nicklas TA, O'Neil CE, et al. (2010) The relationship of breakfast skipping and type of breakfast consumption with nutrient intake and weight status in children and adolescents: the National Health and Nutrition Examination Survey 1999-2006. J Am Diet Assoc 110, 869-878.

8. Sjöberg A, Hallberg L, Höglund D, et al. (2003) Meal pattern, food choice, nutrient intake and lifestyle factors in The Göteborg Adolescence Study. Eur J Clin Nutr 57, 1569-1578.

9. Dubois L, Girard M, Potvin Kent M, et al. (2009) Breakfast skipping is associated with differences in meal patterns, macronutrient intakes and overweight among pre-school children. Public Health Nutr 12, 19-28.

10. Kral TV, Whiteford LM, Heo M, et al. (2011) Effects of eating breakfast compared with skipping breakfast on ratings of appetite and intake at subsequent meals in 8- to 10-y-old children. Am J Clin Nutr 93, 284-291.

11. Leidy HJ \& Racki EM (2010) The addition of a protein-rich breakfast and its effects on acute appetite control and food intake in 'breakfast-skipping' adolescents. Int J Obes (Lond) 34, 1125-1133.

12. Clayton DJ, Barutcu A, Machin C, et al. (2015) Effect of breakfast omission on energy intake and evening exercise performance. Med Sci Sports Exerc 47, 2645-2652.

13. Reeves S, Huber JW, Halsey LG, et al. (2014) Experimental manipulation of breakfast in normal and overweight/obese participants is associated with changes to nutrient and energy intake consumption patterns. Physiol Behav 133, 130-135.

14. Betts JA, Richardson JD, Chowdhury EA, et al. (2014) The causal role of breakfast in energy balance and health: a randomized controlled trial in lean adults. Am J Clin Nutr 100, 539-547.

15. Yoshimura E, Hatamoto Y, Yonekura S, et al. (2017) Skipping breakfast reduces energy intake and physical activity in healthy women who are habitual breakfast eaters: a randomized crossover trial. Physiol Behav 174, 89-94.

16. Chowdhury EA, Richardson JD, Holman GD, et al. (2016) The causal role of breakfast in energy balance and health: a randomized controlled trial in obese adults. Am J Clin Nutr 103, 747-756. 
17. Leidy HJ, Ortinau LC, Douglas SM, et al. (2013) Beneficial effects of a higher-protein breakfast on the appetitive, hormonal, and neural signals controlling energy intake regulation in overweight/obese, 'breakfast-skipping,' late-adolescent girls. $\mathrm{Am} \mathrm{J}$ Clin Nutr 97, 677-688.

18. Leidy HJ, Hoertel HA, Douglas SM, et al. (2015) A high-protein breakfast prevents body fat gain, through reductions in daily intake and hunger, in 'Breakfast skipping' adolescents. Obesity (Silver Spring) 23, 1761-1764.

19. Boushey CJ, Kerr DA, Wright J, et al. (2009) Use of technology in children's dietary assessment. Eur J Clin Nutr 63, Suppl. 1, S50-S57.

20. Martin CK, Nicklas T, Gunturk B, et al. (2014) Measuring food intake with digital photography. J Hum Nutr Diet 27, Suppl. 1, S72-S81.

21. Wang DH, Kogashiwa M \& Kira S (2006) Development of a new instrument for evaluating individuals' dietary intakes. J Am Diet Assoc 106, 1588-1593.

22. Martin CK, Newton RL Jr, Anton SD, et al. (2007) Measurement of children's food intake with digital photography and the effects of second servings upon food intake. Eat Behav 8, 148-156.

23. Atkinson G \& Batterham AM (2015) True and false interindividual differences in the physiological response to an intervention. Exp Physiol 100, 577-588.

24. Janssen I, Katzmarzyk PT, Boyce WF, et al. (2005) Comparison of overweight and obesity prevalence in school-aged youth from 34 countries and their relationships with physical activity and dietary patterns. Obes Rev 6, 123-132.

25. Corder K, van Sluijs EM, Steele RM, et al. (2011) Breakfast consumption and physical activity in British adolescents. Br J Nutr 105, 316-321.

26. Vissers PA, Jones AP, Corder K, et al. (2013) Breakfast consumption and daily physical activity in 9-10-year-old British children. Public Health Nutr 16, 1281-1290.

27. Corder K, van Sluijs EM, Ridgway CL, et al. (2014) Breakfast consumption and physical activity in adolescents: daily associations and hourly patterns. Am J Clin Nutr 99, 361-368.

28. Halsey LG, Huber JW, Low T, et al. (2012) Does consuming breakfast influence activity levels? An experiment into the effect of breakfast consumption on eating habits and energy expenditure. Public Health Nutr 15, 238-245.

29. Todd AS, Street SJ, Ziviani J, et al. (2015) Overweight and obese adolescent girls: the importance of promoting sensible eating and activity behaviors from the start of the adolescent period. Int J Environ Res Public Health 12, 2306-2329.

30. Timlin MT, Pereira MA, Story M, et al. (2008) Breakfast eating and weight change in a 5-year prospective analysis of adolescents: project EAT (Eating Among Teens). Pediatrics 121, 638-645.

31. Cole TJ, Freeman JV \& Preece MA (1995) Body mass index reference curves for the UK, 1990. Arch Dis Child 73, 25-29.

32. McCarthy HD, Jarrett KV, Emmett PM, et al. (2005) Trends in waist circumferences in young British children: a comparative study. Int J Obes (Lond) 29, 157-162.

33. Brooks-Gunn J, Warren MP, Rosso J, et al. (1987) Validity of self-report measures of girls' pubertal status. Child Dev $\mathbf{5 8}$, 829-841.

34. Morris NM \& Udry JR (1980) Validation of a self-administered instrument to assess stage of adolescent development. $J$ Youth Adolesc 9, 271-280.

35. Tanner JM (1962) Growth At Adolescents. Oxford: Blackwell Scientific.

36. Atkinson FS, Foster-Powell K \& Brand-Miller JC (2008) International tables of glycemic index and glycemic load values. Diabetes Care 31, 2281-2283.
37. Wolever TM \& Jenkins DJ (1986) The use of the glycemic index in predicting the blood glucose response to mixed meals. Am J Clin Nutr 43, 167-172.

38. Food Standards Agency (2007) Nutrient and food based guidelines for UK institutions. http://www.food.gov.uk/ multimedia/pdfs/nutrientinstitution.pdf (accessed March 2017).

39. Timlin MT \& Pereira MA (2007) Breakfast frequency and quality in the etiology of adult obesity and chronic diseases. Nutr Rev 65, 268-281.

40. Scientific Advisory Committee on Nutrition (2011) Dietary Reference Values for Energy. London: TSO, Public Health England.

41. Foster E, Adamson A, Hawkins A, et al. (2010) Young Person's Food Atlas - Secondary. London: Food Standards Agency.

42. Foster E \& Adamson AJ (2012) Development and validation of the Young Person's Food Atlas. Proc Nutr Soc 71, 195.

43. Foster E, Hawkins A, Barton KL, et al. (2017) Development of food photographs for use with children aged 18 months to 16 years: comparison against weighed food diaries - The Young Person's Food Atlas (UK). PLOS ONE 15, 12:e0169084.

44. NHS Choices (2015) 5 A DAY: portion guide. www.nhs.uk/ Livewell/5ADAY/Documents/Downloads/5ADAY_portion_ guide.pdf (accessed March 2017).

45. Bland JM \& Altman DG (1986) Statistical methods for assessing agreement between two methods of clinical measurement. Lancet 1, 307-310.

46. Wang YC, Gortmaker SL, Sobol AM, et al. (2006) Estimating the energy gap among US children: a counterfactual approach. Pediatrics 118, 1721-1733.

47. Phillips LR, Parfitt G \& Rowlands AV (2013) Calibration of the GENEA accelerometer for assessment of physical activity intensity in children. J Sci Med Sport 16, 124-128.

48. Rowlands AV, Rennie K, Kozarski R, et al. (2014) Children's physical activity assessed with wrist- and hip-worn accelerometers. Med Sci Sports Exerc 46, 2308-2316.

49. Cohen J (1988) Statistical Power Analysis for the Behavioral Sciences, 2nd ed. Hillsdale, NJ: Lawrence Erlbaum Associates.

50. Schmitz K, Treuth MS, McMurray R, et al. (2005) Predicting energy expenditure from accelerometry counts in adolescent girls. Med Sci Sport Exerc 37, 155-161.

51. Treuth MS, Catellier DJ, Schmitz KH, et al. (2007) Weekend and weekday patterns of physical activity in overweight and normal-weight adolescent girls. Obesity (Silver Spring) 15, 1782-1788

52. Chowdhury EA, Richardson JD, Tsintzas K, et al. (2015) Carbohydrate-rich breakfast attenuates glycaemic, insulinaemic and ghrelin response to ad libitum lunch relative to morning fasting in lean adults. Br J Nutr 114, 98-107.

53. Shaw ME (1998) Adolescent breakfast skipping: an Australian study. Adolescence 33, 851-861.

54. Warren JM, Henry CJ \& Simonite V (2003) Low glycemic index breakfasts and reduced food intake in preadolescent children. Pediatrics 112, 414-419.

55. Ball SD, Keller KR, Moyer-Mileur LJ, et al. (2003) Prolongation of satiety after low versus moderately high glycemic index meals in obese adolescents. Pediatrics 111, 488-494.

56. Bornet FR, Jardy-Gennetier AE, Jacquet N, et al. (2007) Glycaemic response to foods: impact on satiety and long-term weight regulation. Appetite 49, 535-553.

57. Sun FH, Li C, Zhang YJ, et al. (2016) Effect of glycemic index of breakfast on energy intake at subsequent meal among healthy people: a meta-analysis. Nutrients $\mathbf{8}, 37-51$.

58. Macdiarmid J \& Blundell J (1998) Assessing dietary intake: who, what and why of under-reporting. Nutr Res Rev 11, 231-253.

59. Burrows TL, Martin RJ \& Collins CE (2010) A systematic review of the validity of dietary assessment methods in children when 
compared with the method of doubly labeled water. J Am Diet Assoc 110, 1501-1510.

60. Livingstone MB, Robson PJ \& Wallace JM (2004) Issues in dietary intake assessment of children and adolescents. $\mathrm{Br} J$ Nutr 92, Suppl. 2, S213-S222.

61. Small L, Sidora-Arcoleo K, Vaughan L, et al. (2009) Validity and reliability of photographic diet diaries for assessing dietary intake among young children. ICAN 1, 27-36.

62. Tarasuk V \& Beaton GH (1991) The nature and individuality of within-subject variation in energy intake. Am J Clin Nutr 54, 464-470.

63. Carlsohn A, Fusch G \& Scharhag-Rosenberger F (2011) Validity and reliability of athletes' self-recorded food intake determined by doubly-labelled water. Med Sci Sports Exerc 43, 503-504.

64. Tremblay A, Sévigny J, Leblanc C, et al. (1983) The reproducibility of a three-day dietary record. Nutr Res 3, 819-830.

65. Toeller M, Buyken A, Heitkamp G, et al. (1997) Repeatability of three-day dietary records in the EURODIAB IDDM Complications Study. Eur J Clin Nutr 51, 74-80.
66. Chaput JP, Jomphe-Tremblay S, Lafrenière J, et al. (2016) Reliability of a food menu to measure energy and macronutrient intake in adolescents. Eur J Clin Nutr 70, 104-108.

67. Arora M, Nazar GP, Gupta VK, et al. (2012) Association of breakfast intake with obesity, dietary and physical activity behavior among urban school-aged adolescents in Delhi, India: results of a cross-sectional study. BMC Public Health 12, 881-893.

68. Utter J, Scragg R, Mhurchu CN, et al. (2007) At-home breakfast consumption among New Zealand children: associations with body mass index and related nutrition behaviors. I Am Diet Assoc 107, 570-576.

69. Bingham S (1987) The dietary assessment of individuals; methods, accuracy, new techniques and recommendations. Nut Abstracts Rev 57, 705-742.

70. Hjorth MF, Damsgaard CT, Michaelsen KF, et al. (2015) Markers of metabolic health in children differ between weekdays - the result of unhealthier weekend behavior. Obesity (Silver Spring) 23, 733-736. 